\title{
Actividad antioxidante de esteroles y ácidos orgánicos naturales
}

\author{
Por R. Maestro-Durán y R. Borja-Padilla \\ Instituto de la Grasa y sus Derivados (C.S.I.C.). Apartado 1078. 41012 - Sevilla.
}

\section{RESUMEN}

Actividad antioxidante de esteroles y ácidos orgánicos naturales.

Continuando el estudio de los antioxidantes naturales, se incluyen en este trabajo los esteroles y los ácidos orgánicos no estudiados en los trabajos anteriores (ácidos fenólicos, en "Actividad antioxidante de los compuestos fenólicos»: ácido ascórbico, en «Actividad antioxidante de las vitaminas $C$ y $D$ y de la provitamina $A$ », aminoácidos, en «Actividad antioxidante de compuestos naturales nitrogenados").

Los ácidos orgánicos cuya actividad como antioxidantes se estudia en este trabajo son: cítrico, málico, láctico, fumárico, sórbico, tartárico, kójico, fítico y furoicos. Se discuten sus mecanismos de reacción en cadena de los radicales prooxidantes, bien por secuestro de estos radicales.

Finalmente, se citan las patentes registradas en los últimos años por estos compuestos o sus derivados como antioxidantes o como sinergistas con otros productos.

PALABRAS-CLAVE: Acido orgánico - Antioxidante (actividad) - Esterol Información (artículo).

\section{SUMMARY}

\section{Antioxidant activity of natural sterols and organic acids.}

This paper deals with antioxidant activity of sterols and natural organic acids not covered on previous papers. The paper «Antioxidant activity of phenolic compounds" covered phenolic acids; the second one "Antioxidant activity of vitamin C, D and provitamin A», ascorbic acid, and the third "Antioxidant activity of natural nitrogenous compounds", aminoacids.

The acids studied here included citric, malic, lactic, fumaric, tartaric, kojic (5-hydroxy-2-[hydroxymethyl- $\gamma$-pyrone], phytic (inositol-hexaphosphoric acid) and furoic acids.

The reaction mechanisms, either as inhibitors of the chain reaction of prooxidant radicals or by sequestration of free radicals was discussed.

Finally, a list of recent patents of this compounds and their derivatives as antioxidants or synergists was included.

KEY-WORDS: Antioxidant (activity) - Information (paper) - Organic acid - Sterol.

\section{INTRODUCCION}

En el presente trabajo se recogen los estudios sobre el carácter antioxidante o sinergista con otros antioxidantes de los esteroles y de los ácidos orgánicos no estudiados en trabajos anteriores, es decir, a excepción de los fenólicos, el ascórbico y los aminoácidos.

\section{ESTEROLES}

Se sabe desde los años sesenta que los 4-metil-esteroles, aislados de la fracción insaponificable de los aceites de germen de trigo y de maíz inhiben la oxidación del ácido linoleico en los aceites de freír. También el insaponificable del aceite de Vernonia Anthelmíntica contiene una cantidad relativamente alta de esteroles que protegen de la oxidación al aceite de cártamo (Fioriti, 1971). Se ha llegado a la conclusión (Sims, 1972) de que la cadena de isofucosterol en $\mathrm{C}-17$ era la parte activa de la molécula, ya que el ergosterol, estigmasterol, $\beta$-sitosterol y colesterol muestran un cierto carácter prooxidante.

Aunque el esterol más importante del aceite de oliva es el $\beta$-sitosterol, se han encontrado aceites con más del $9 \%$ de $\Delta^{5}$-avenasterol (Boskou, 1975).

El ciclostadienol ( $\alpha$-sitosterol), que es un 4 -metil-esterol, es también un eficaz antioxidante. Este hecho puede explicarse por la hipótesis de que tanto los 4-metil como los 17 etiliden esteroles pueden reaccionar rápidamente con los radicales libres de los lípidos para dar otros radicales, relativamente estables, que son los antioxidantes, ya que interrumpen la reacción en cadena de la autooxidación de los lípidos. De esta manera, los esteroles con un grupo etiliden en la cadena lateral son más eficaces como antioxidantes, pero su eficiencia mayor puede alcanzarse con la presencia y la posición de uno o más dobles enlaces endocíclicos. El vernosterol tiene carácter antioxidante por la rápida formación de radicales libres en $\mathrm{C}-29$, pero la formación, más lenta, de radicales libres en C-11 o C-16, que se estabilizarían por deslocalización de los dos dobles enlaces, también contribuiría a la actividad antioxidante del vernosterol. De hecho, es más efectivo que los esteroles con un solo doble enlace endocíclico, como el fucosterol. Los mecanismos de la actividad antioxidante del $\Delta^{5}$ - avenasterol fueron estudiados por Willianson (1988).

Se probaron con aceite de algodón calentado a $80^{\circ}$ los 5-metil esteroles aislados de aceite crudo de girasol por saponificación y esterificados con cloruro de estearilo, al tiempo que se hacía una prueba paralela con los 4-metil esteroles libres (Blekas, 1986). Ambos, esteroles y ésteres de esteroles, fueron igualmente efectivos para evitar la polimerización oxidativa del aceite calentado.

Hay registrada una patente para cosméticos blanquea- 
dores de la piel que inhiben la formación de melanina, conteniendo, además de otros ingredientes, palmitato de fitosterol (Takeuchi, 1989).

\section{ACIDOS ORGANICOS}

\subsection{Acido cítrico}

El ácido cítrico es un antioxidante muy eficaz por su capacidad para inactivar trazas metálicas. En un test con aceite de soja a $100^{\circ} \mathrm{C}$ en un método con oxígeno activo, al cabo de 10 horas, el índice de peróxido fue de 62 en el aceite control, de 28 con $0,01 \%$ de ácido cítrico y de 18 con 0,005 de ácido cítrico $+0,15 \%$ de sorbitol.

Junto a otros ácidos orgánicos (láctico, tartárico y málico), el ácido cítrico ha sido empleado para alargar el tiempo de conservación de productos derivados de pescados (Priebe, 1980).

Aunque el BHT se ha mostrado ineficaz para proteger, a concentración $\leq 500 \mathrm{ppm}$, de la oxidación a los ácidos grasos de manteca de cerdo, éstos muestran una buena estabilidad con 1000 ppm de una mezcla BHT-ácido cítrico (Erdely, 1985).

Se ha empleado con eficacia el ácido cítrico para evitar el enranciamiento de ácidos grasos destilados de sebo vacuno (Grompone, 1987).

Por el contrario, el ácido cítrico se comporta como ligeramente prooxidante para los lípidos de pescado (menhaden, Brevoortia patronus) congelado a $-20^{\circ} \mathrm{C}$ (Hwang, 1988).

Se han registrado en los últimos años las siguientes patentes para antioxidantes en cuya formulación figura el ácido cítrico o alguna de sus sales:

Como antioxidante para procesar champiñones (Knehneromyces nameko) (Ueno, 1974), y polifosfato (0,5-0,01 $\%$ de cada uno), conservante de pescado envasado en latas (Skrodzki, 1982), antioxidante de aceites y grasas (Tanabe, 1982), conservante de vegetales, ensaladas y frutas troceadas (Organogen Medizinisch Germany, 1987), desodorante de residuos sólidos (Ichiji, 1987 a), antioxidante y desodorante para hospitales y cocinas (Ichiji, 1987 b), inhibidor de la oxidación de compuestos de hierro solubles en agua (Ichiji, 1987 c), antioxidante para alimentos, cosméticos y productos farmacéuticos (Iwai, 1988) y para prevenir la pérdida de color en alimentos procesados (Fukazawa, 1990 a).

\subsection{Acido málico}

El efecto retardador del ácido málico de la degradación oxidativa de los lípidos es bien conocido, tanto en usos no alimentarios, como barnices y sustancias selladoras, espuma de poliuretano y estireno, como en la estabilización de aceites y grasas comestibles, como aceite de soja, linoleato de metilo en un sistema acuoso o aceite de oliva al que se había añadido oleato de cobre y calentado a $50^{\circ} \mathrm{C}$.

El ácido málico actúa como sinergista con catequinas extraídas de hojas verdes de te en la protección de manteca de cerdo y de aceites para ensalada (Matsuzaki, 1984). También protege el flavor de huevos duros, como se demostró por el método del panel test (Sheldon, 1986).

Preparados en cuya composición forma parte el ácido málico han sido patentados por Tanabe (1982), ya citado, para alimentos procesados por Takeda Ind. Lim. (1983), como desoxigenante en recipientes cerrados, por Kawasaki Kasei Chemicals (1983), como antioxidante y estabilizador al calor de bisfenoles procesados (Neil, 1988).

\subsection{Acido láctico}

El ácido láctico ha sido empleado como antioxidante a una concentración de $1,5 \%+1,5 \%$ de etanol para el sedimento que queda de la salsa de soja cruda después de pasteurizarla. Es una torta que puede usarse en alimentos procesados como un sustitutivo de la salsa de soja. También, a la concentración de 0,2-0,3\% con respecto a la harina, se ha empleado para evitar el enranciamiento y la contaminación por microorganismos de pan o productos horneados (Mogilko, 1978).

Pulverizando con un spray al 3\% de ácido láctico en agua canales de buey previamente enfriadas, se inhibe el crecimiento bacteriano y se aumenta hasta 60 veces el tiempo que pueden conservarse en frío sin deteriorarse (Wichlack, 1986).

Se han citado ya patentes de preparados en cuya composición forma parte el ácido láctico: (Ueno, 1974), (Tanabe, 1982), (Takeda, 1983), (Kawasaki, 1983), (Sheldon, 1986), (Crawford, 1985) y (Neil, 1988). Otras patentes para el ácido láctico o sus sales son como preservativos para perfumes, cosméticos y productos químicos para el hogar (Kruchinin, 1980), lactato cálcico y quitosano, como conservante en polvo para alimentos soluble en agua (Watanabe, 1987), como sinergista de conservantes de alimentos constituidos por partículas pequeñas, junto con polioles (glicerol, propilen- glicol o butanodiol), (Ramet, 1987) y como estabilizador de aductos de óxidos de alquilenos (Yokohama, 1988).

\subsection{Acido fumárico}

Se han registrado dos patentes de cuya formulación forma parte el ácido fumárico: mezclado con $\varepsilon$-polysina 0 sus sales, como conservante de alimentos cárnicos (Morita, 1987) y como estabilizador de vitaminas premezcladas a las que se añade un $35-40 \%$ de ácido fumárico (Pelevin, 1988).

\subsection{Acido sórbico}

El ácido sórbico se ha empleado como un protector del color de zumos de frutas, como manzanas, ya que los carotenoides pierden el color cuando se oxidan. Esta autooxidación, promovida por radicales libres, como ter-butilhidroperóxidos o hidroperóxidos de lípidos. Se ha 
empleado también para proteger a la vitamina A en preparados farmacéuticos (Van Dooren, 1985).

Además de antioxidante, el ácido sórbico está reconocido como un potente fungistático en los alimentos, a los que protege también del enranciamiento. Además, el sorbato potásico inhibe el crecimiento de levaduras (Woolford, 1975) y ha sido empleado como ingrediente de aditivos comerciales en silos (Alli, 1982). El ácido sórbico (0,90 g. $\left.\mathrm{kg}^{-1}\right)+$ sorbato potásico $\left(0,90 \mathrm{~g} \cdot \mathrm{kg}^{-1}\right)$ han sido aplicados para proteger de la oxidación y del ataque por microorganismos a plantas de maíz y alfalfa ensiladas para piensos de animales (Alli, 1985).

Existen dos patentes, ya citadas, para un antioxidante en cuya formulación figura el sorbato sódico (Ichiji, 1987 a) y para conservar pescado conteniendo fosfatos (Crawford, 1985).

\subsection{Acido tartárico}

Ha sido empleado para aumentar el tiempo de conservación de filetes de arenque preparados como sucedáneo de salmón (Priebe, 1980). También actúa como conservante de pescados mejor que el ácido fosfórico (Crawford, 1985).

Una patente, también citada (Matsuzaki, 1984), emplea ácido tartárico como sinergista con compuestos fenólicos extraídos de hojas verdes de té en evitar la oxidación de manteca de cerdo.

\subsection{Acido kójico}

En realidad, no se trata de un ácido orgánico, sino de una lactona, 5-hidroxi-2-(hidroximetil)-5-pirona. Es una $\gamma$ pirona natural producida por microorganismos, mohos. Fue aislado en cultivos de Aspergillus oryzae y posteriormente de otras especies de Penicillium, y de Aspergillus, y en frutos de Ficus.

Es un poderoso antioxidante porque secuestra iones metálicos que inducen la reacción en cadena de radicales libres de la autooxidación de los lípidos. Se han realizado numerosos trabajos sobre esta acción secuestrante.

En lo referente al empleo del ácido kójico como antioxidante para alimentos, ha demostrado una gran efectividad como antioxidante del aceite de soja (Yi, 1982). Se ha empleado también como antioxidante para tallarines y fideos crudos, porque evita, a la concentración de $3,3.10^{-5} \mathrm{M}$ la acción de la tirosinasa del salvado de trigo, que puede dar manchas no deseables (Uchino, 1988). Otros antioxidantes, como BHA, BHT, $\alpha$-tocoferol o ácido ascórbico no son efectivos para evitar la formación de tales manchas.

Al igual que otros antioxidantes secuestradores de radicales libres, el ácido kójico evita el enranciamiento de carne de buey (St. Angelo, 1988). Como consecuencia de esta actividad antioxidante del ácido kójico existen numerosísimas patentes sobre preparados en cuya composición entra dicho ácido: como antioxidante para grasas, aceites, alimentos relacionados con ellos, medicinas y cosméticos (Sansho Seiyaku C. Ltd., 1981), del aceite de maíz (Sansei
Pharmaceutical Co. Ltd. 1983), como conservante de alimentos frescos, como champiñones, bañándolos o rociándolos con solución acuosa de ácido kójico (Uchino, 1987), en películas $u$ hojas para envolver flores cortadas, frutas y vegetales o lechugas frescas envueltas en Styrofilm al que se había añadido $300 \mathrm{mg}$ de ácido kójico/m2 (Kato, 1987), derivados del ácido kójico como antioxidantes y decolorantes de cosméticos por tener mayor actividad inhibidora de la tirosinasa (Nakajima, 1984), en cosméticos para blanquear la piel, junto con vitamina $E$ porque inhibe la melanogénesis conteniendo ácido kójico (Oyama, 1987), ácido kójico y ácidos grasos insaturados desde $\mathrm{C}_{18-22}$ con $\geq 2$ dobles enlaces (Ando, 1989), ácido kójico y otros sinergistas inhibidores de la tirosinasa (Oyama, 1989). Se han registrado también patentes de productos conteniendo ácido kójico para evitar la pérdida de color de alimentos procesados, como jamón cocido, salchichas, huevas de bacalao (Fukazawa, 1990 a). El jamón y las salchichas mantienen su color, y las huevas de bacalao adquieren un color amarillo-rojizo que permanece durante más de catorce días. Camarones bañados en una solución acuosa de $0,6 \mathrm{~g} / \mathrm{L}$, a una temperatura de $5^{\circ} \mathrm{C}$ mantienen el color durante cinco días (Fukazawa, 1990 b).

\subsection{Acido fítico}

El ácido fítico (inositol hexa-fosfórico), es un constituyente muy abundante en las plantas, comprendiendo del 1 al $6 \%$ en peso de legumbres, cereales, semillas oleaginosas, pólenes y nueces, y entre el 60 y el $90 \%$ del fósforo total (Cheryan, 1980). Generalmente se encuentra en forma de sales de magnesio y de potasio (fitinas) en determinadas partes de las semillas, como la capa de aleurona del trigo o el arroz (Tanaka, 1974), en el germen de maíz (O'Dell, 1972) o en glóbulos tipo cristaloide de muchas semillas oleaginosas (Lui, 1967). El ácido fítico puede obtenerse puro y a bajo costo a partir de salvado de varias semillas, que lo contienen entre un 5 y $6 \%$, extrayéndolo con $\mathrm{ClH}$ diluido a temperatura ambiente, precipitándolo por adición de $\mathrm{FeCl}_{3}$, bicarbonatos, $\mathrm{KOH}, \mathrm{NaOH}, \mathrm{NH}_{4} \mathrm{OH}$, $\mathrm{Ca}(\mathrm{OH})_{2}, \mathrm{Mg}(\mathrm{OH})_{2}$ o alcohol y purificándolo por técnicas químicas habituales (Graf, 1983 b). También puede obtenerse de las aguas residuales de obtención de proteínas comestibles de semilla de algodón.

La estructura tan peculiar del ácido fítico hace de él un agente secuestrante importantísimo. Precipita cuantitativamente el $\mathrm{Fe}^{3+}$ (Vohra, 1965) a pH bajo y a pHs intermedios y altos forma también complejos insolubles con otros cationes polivalentes (Maddaiah, 1964).

El ácido fítico es un antioxidante muy bueno. A diferencia de la mayor parte de los quelantes, el complejo que forma con el $\mathrm{Fe}^{3+}$ impide a éste catalizar la formación de radicales libres. Inhibe marcadamente la oxidación de la vitamina C. La acción sinergista del ácido fítico con mezclas de tocoferoles también se ha mostrado útil para proteger de la autooxidación al oleato de metilo y otros lípidos.

El ácido fítico proporciona un estado de oxidación exclusivamente trivalente del hierro y bloquea así el ciclo de oxidación-reducción $\mathrm{Fe}^{3+}-\mathrm{Fe}^{2+}$ necesario para muchas 
reacciones de oxidación. Además, concentraciones altas de ácido fítico previenen el pardeamiento y la putrefacción de frutas y verduras porque inhibe la polifenoloxidasa. Estas observaciones indican que el fitato desempeña una importante función antioxidante en las semillas durante la dormancia (Graf, 1987).

Las propiedades del ácido fítico como secuestrador del $\mathrm{Fe}^{3+}$ ya citadas convierten a este compuesto en un antioxidante único y versátil. Previene de la oxidación al aceite de soja (Nishina, 1987) y estabiliza otros lípidos evitando el enranciamiento (Yamamota, 1973). Añadido a los alimentos, protege a la carne (Tanaka, 1980), a pastas de harina de pescado (Tanikawa, 1972), fideos cocinados (Wakita, 1977), pan, ensalada, pescado (Mizuta, 1974) y estabiliza colorantes, tanto naturales como artificiales. Preserva, por ejemplo, al caviar (Uekoki, 1977), a vegetales frescos (Jato, 1980), a sardinillas desecadas (Ajinomoto Co. Ltd. 1980) y a gambas (Zhang, 1986), ya que inhibe la actividad de la tirosinasa.

También se ha estudiado la influencia del ácido fítico en la estabilidad del ácido ascórbico en soluciones acuosas y en emulsiones de aceite en agua. En ambos sistemas, el ácido fítico a concentración $1 \mathrm{mM}$ proporciona protección contra los daños oxidativos y aumenta cuatro veces la estabilidad de la emulsión (Empson, 1991).

\subsection{Acidos furoicos}

Los ácidos grasos con un anillo furano están ampliamente repartidos en los lípidos de peces, sobre todo de los machos. Se descubrieron por primera vez en el lucio (Glass, 1974), donde se encuentran en todos los tejidos, pero sobre todo en el hígado y testículos. De momento no pudieron establecer las estructuras de estos ácidos grasos, aunque comprobaron que tenían tres átomos de oxígeno, que eran más resistentes a la oxidación que los ácidos insaturados normales y que no formaban aductos con urea. Un año más tarde fueron identificados como ácidos furoicos por el mismo autor (Glass, 1975), quien comprobó también que su contenido era máximo en los órganos sexuales y mínimo en el hígado durante la época de la freza. Posteriormente se encontraron en hígados de peces, como bacalao (Scrimgeour, 1977), perca, salmón, arenque, gobio, capellina, abadejo y caballa (Gunstone, 1978). Se han detectado también ácidos furoicos en otros tipos de animales distintos de los peces marinos o de agua dulce. En el cangrejo se encuentran como ésteres del colesterol en el hepatopáncreas (Ishii, 1988) y en los fosfolípidos (Ishii, 1989), en moluscos como el pulpo (Gunstone, 1978), en crustáceos como la langosta, en insectos, en batracios, en reptiles (Ishii, 1988), en mamíferos como la vaca e incluso en el hombre (Puchta, 1988). En plantas también es frecuente la existencia de ácidos furoicos: en látex de Hevea brasiliensis (Hasna, 1978), en césped, diente de león, trébol, hojas de abedul, cebolleta, trigo, arroz, patata (hojas y tubérculos), col, naranja, limón, fresa (hojas y fruto), champiñones, levaduras y algas (Hannemann, 1989).

Hay una evidencia total de que estos derivados del furano son secuestradores muy efectivos del oxígeno sin- gulete, pero se conoce poco de su capacidad para atrapar radicales libres (Noguchi, 1977). Se ha estudiado también la actividad antioxidante de los furanos sustituidos con ácidos grasos en la oxidación de ácido linoleico en buffer de fosfato de pH 6,9 en la oscuridad (Okada, 1990). Los furanos tetraalquil sustituidos inhibieron completamente la oxidación. Los trialquil sustituidos también son antioxidantes, aunque su eficacia es la mitad que la de los tetraalquil sustituidos. Los dialquil sustituidos no mostraron actividad antioxidante, de lo que se deduce que el poder antioxidante depende del número de sustituyentes del anillo de furano. Los furanos tetrasustituidos se comportaron como secuestradores del radical formado en la oxidación del ácido linoleico, rompiendo la reacción en cadena.

\section{BIBLIOGRAFIA}

Ajinomoto Co. Inc. (1980).- «Dried sardine fingerling preservation with phytin".-Jpn. Kokai Tokkyo Koho JP 80 68,259.

Ando, H., Hashimoto, A., Shimizu, M., Kato, H. e Osaza, Y. (1989).-«Skinlightening cosmetic containing unsatured fatty acids».-Jpn. Kokai Tokkyo Koho JP 01,186,811.

Blekas, G. y Boskov, D. (1986).-«Effect of esterified sterols on the stability of heated oils».-Dev. Food Sci. 1986, 641-645.

Boskou, D. y Morton, D. (1975).-«Changes in the sterol composition of olive oil on heating".-J. Sci. Food Agric. 26, 1149-1153.

Cheryan, M. (1980).-«Phytic acid interactions in food systems".-Crit. Rev. Food Sci. Nutr. 13, 297-355. C.A. 94, 2890t.

Crawford, J.C. (1985).-«Fish preservative containing phosphates».-U.S. Patent US 4,517,208

Empson, K.L., Labuza, T.P. y Graf, E. (1991).-«Phytic acid as a food antioxidant».-J. Food Sci. 56, 560-563.

Erdelyi, A. (1985).-«Autoxidation of fatty acids produced from natural fat and inhibition of the oxidative rancidity".-Dev. Food Sci. 11, 639-648.

Erdman, J.W. (1979).-«Oilseed phytates: nutritional implications».-J. Am. Oil Chemists' Soc. 56, 736-744.

Fioriti, J.A., Kanuk, M.J. y Sims, R.J. (1971).-«The unsaponifiables of Vernonia Anthelmintica seed oil».-J. Am. Oil Chemists' Soc. 48, 240-244.

Foote, C.S. (1976).-«Free radicals in Biology».-Vol. 2, p. 99.-Ed. Pryce, W.A.-Academic Press, Nueva York.

Fukazawa, R., Ito, T., Matsuda, Y., Wakaba, Y., Ashi, H. y Natori, Y. (1990 a).-«Color-fading preventing agents containing kojic acid and method for prevention of color-fading for processed foods".-Jpn. Kokai Tokkyo Koho JP 69,156.

Fukazawa, R., Wakabayashi, H. y Natori, Y (1990 b).-«Kojic acid for prevention of color fading of foods".-Jpn. Kokai Tokkyo Koho JP 02 31,661.

Glass, R.L., Krick, T.P. y Eckhardt, A.E. (1974).-«New series of fatty acids in northern pike (Esox lucius)».-Lipids 9, 1004-1008.

Glass, R.L., Krick, T.P., Sand, D.M., Rahn, C.H. y Schleck, H. (1975).«Furanoic fatty acids from fish lipids».-Lipids 10, 695-702.

Graf, E., Empson, K.L. y Eaton, J.W. (1987).-«Phytic acid. A natural antioxidant".-J. Biol. Chem. 262, 11647-11650.

Grompone, M.A. (1987).-«Enranciamiento de ácidos grasos destilados de sebo vacuno".-Grasas y Aceites 38, 375-378

Gunstone, F.D., Wijesundera, R.C. y Scringeur, C.M. (1978).-«The component acids of lipids from marine and freshwater species with special reference to furan-containing acids".-J. Sci. Food Agr. 29, 539-550.

Hannemann, K., Puchta, V., Simon, E., Ziegler, H., Ziegler, C. y Spiteller, G. (1989).-«The common ocurrence of furan fatty acids in plants».-Lipids 24, 296-298

Harland, B.F. y Harland, J. (1980).- «Fermentative reduction of phytate in rye, white and whole wheat breads".-Cereal Chem. 57, 226-229.

Hasna, H. y Subramaniam, A. (1978).-«The ocurrence of a furanoid fatty acid in Hevea brasiliensis latex".-Lipids 13, 905-907.

Hwang, K.T. y Regestein, J.M. (1988).-«Protection of menhaden mince lipids from rancidity during frozen storage".-J. Food Sci. 54, 1120-1124.

Ichiji, T. (1987 a).-«pH adjustable antioxidized deodorants for waste solids».Jpn. Kokai Tokkyo Koho, JP 62,283,186.

Ichiji, T. (1987 b).-«Antioxidant and deodorant composition».-Jpn. Kokai Tokkyo Koho, JP 62,257,982. 
Ichiji, T. y Nakamura, K. (1987 c).-«Oxidation inhibition for aqueous iron compounds".-Jpn. Kokai Tokkyo Koho, JP 62,176,932.

Ishii, K., Okajima, H., Koyamatsu, T., Okada, Y. y Watanabe, H. (1988)."The composition of furan fatty acids in the crayfish».-Lipids 23, 694695.

Iwai, Y., Sano, T., Kashimata, M. y Kaneka, M. (1988).-«Antioxidation emulsion for foods, cosmetic and pharmaceuticals».-Jpn. Kokai Tokkyo Koho JP 63, 135, 483

Jato, M. (1980).-«Fresh vegetable preservation».- Jpn. Kokai Tokkyo Koho JP 79 24,068.

Kato, Y., Fukazawa, R., Wakabayashi, H., Sato, M. y Natori, Y. (1987).«Packaging films or sheets for cut flowers, fruits and vegetables».-Jpn. Kokai Tokkyo Koho JP 01 06,034.

Kawasaki Kasei Chemicals Ltd. (1983).-«Deoxidizing agent».-Jpn. Kokai Tokkyo Koho JP 60 94,137.

Kontoghiorghes, G.J., Jackson, M.J. y Lunec, J. (1986).-«In vitro screening of iron chelators using models of free damage».-Free Radical Res. Comm. 2, 115-124.-C.A. 106, 61190r.

Krucinin, V.A., Nechesnyuk, G.P., Volokhina, M.I., Bichman, B.I., Urinovich, E.M. y Logunov, Yu. V. (1980).-«Preservatives for perfumes, cosmetics and household chemicals".-U.S.S.R. SU 1,088,727.

Lui, N.S.T. y Altschul, A.M. (1967).-«Isolation of globoids from cottonseed aleurone grain".-Arch. Biochem. Biophys. 121, 678-684.

Maddaiah, A.A., Kurnik, B., Hulett, B.J. y Red, B.L. (1964).-«Nature of intestinal phytase activity».-Proc Soc. Exptl. Biol. Med. 115, 1054-1057.-C.A. 65, 3491b.

Maestro-Durán, R. y Borja-Padilla, R. (1993 a).-«Actividad antioxidante de los compuestos fenólicos".-Grasas y Aceites 44, 101-106.

Maestro-Durán, R. y Borja-Padilla, R. (1993 b).-«Actividad antioxidante de las vitaminas $C$ y $E$ y de la provitamina $A$ »-Grasas y Aceites 44, 107111.

Maestro-Durán, R. y Borja-Padilla, R. (1993 c).-«Actividad antioxidante de compuestos nitrogenados".-Grasas y Aceites 44, 204-207.

Matsuzaki, T. y Hara, Y. (1984).- «Antioxidative activity of tea leaf catechins".- Nippon Nogei Kagaku Kaishi 59. 129-134.

Mizuta, K., Toyoda, Y., Kusama, I., Kakizawa, T. y Kumekawa, K. (1974)."Glicine as a food preservative».- Jpn. Kokai Tokkyo Koho JP 74 13,343.

Mogilko, I.P. (1978).- «Prevention of microbiological spoilage of baked goods".- Khlebopk, Konditer Prom-st. 2. 40-41.- C.A. 88. 150913f.

Morita, Y., Kurokawa, Y. y Fujii, M. (1987).-«Preservative for foods».- Jpn. Kokai Tokkyo Koho JP $6258,975$.

Nakajima, K. y Higa, Y. (1984).- «Kojic acid derivatives».- Jpn. Kokai Tokkyo Koho JP 60,233,071.

Neil, L.E. Jr. y Gormanos, T.J. (1988).-«Antioxidants and heat stabilizers for bisphenols".- U.S. US 4,894,486.

Neil, L.E., Jr. y Gormanos, T.J. (1990).-«Antioxidants and heat stabilizers for bisphenols».-U.S. US 4,894,486.

Nishina, A. e Ito, M. (1987).-«Antioxidant composition for oils and fats».-Jpn. Kokai Tokkyo Koho $6248,788$.

Noguchi, T., Takayama, K. y Nakano, M. (1977).-"Conversion of 2,5dimethylfuran to 2-hydroxy-5 hydroperoxy-2,5-dimethylfuran, a true ${ }^{1} \mathrm{O}_{2}$ derived reaction in aqueous ${ }^{1} \mathrm{O}_{2}$ generating systems".-Biochem. Biophys. Res. Commun. 78, 418-423.

Nonhebel, D.C. y Walton, J.C. (1974).-«Free-radical chemistry»--Cambridge University Press, Cambridge.

O'Dell, B.L., DeBoland, A.R. y Koiryohann, S.R. (1972).-«Distribution of phytate and nutritionally important elements among the morphological components of cereal grains".-J. Agric. Food Chem. 20, 718-721.

Okada, Y., Okajima, H., Konishi, M., Terauchi, M., I. Min, L. y Watanabe, H. (1990).- «Antioxidant effect of naturally occurring furan fatty acids on oxidation of linoleic acid in aqueous dispersion».-J. Am. Oil Chemists' Soc. 67, 858-862.

Organogen Medizinisch-Molekularbiologische Forschunsgesellschaft. Ger Offen. DE 3,624,035. Oyama, Y. (1987).-«Cosmetics containing kojic acid and vitamin $E$ for the inhibition of melanin formation".-Jpn. Koka Tokkyo Koho JP 62,178,506.

Oyama, Y. (1987).-«Cosmetic containings kojic acid and vitamin E for the inhibition of melanin formation".-Jpn. Kokai Tokkyo Koho JP 62, 178 506.

Oyama, Y. (1989).-«Cosmetics with skin-lightening properties containing kojic acid derivatives and melanin synthesis-inhibiting compounds".-Eur. Pat. Appl. EP 308,919.

Pelevin, A.D., Buzlama, V.S., Alekhina, S.K. y Shentsova, E.S. (1988).-
"Method of stabilizing vitamins with fumaricacid».-U.S.S.R. SU $1,395,270$.

Puchta, V., Spiteller, G. y Weidinger, H. (1988).-«F acids: a previously unknown component of the phospholipids of human blood".-Liebigs Ann. Chem. 1988, 25-28.

Ramet, J.P. (1987).-«Preservatives for foods made of small particles».-Fr. Demande FR 2,610,795

Sansei Pharmaceutical Co. Ltd. (1983).-«Antioxidant composition for oils and fats".-Jpn. Kokai Tokkyo Koho JP 58,160,399.

St. Angelo, A.J., Vercellotti, J.R., Dupuy, H.P. y Spanier, A.M. (1988)."Assessment of beef flavor quality: a multidisciplinar approach".-Food Technol. 42, 133-138.

Sansho Seiyaki Co. Ltd. (1981).-«Kojic acid as antioxidant».-Jpn. Kokai Tokkyo Koho JP 81 24,484.

Scrimgeour, C.M. (1977).-«Quantitative analysis of furanoid acids in crude and refined cod liver oil».-J. Am. Oil Chemists' Soc 54, 210-211.

Sheldon, B.W. (1986).-«Influence of three organic acids on the quality characteristics of hard-cooked eggs".-Poult. Sci. 65, 294-301.-C.A. 105, 132394c.

Sims, R.J., Fioriti, J.A. y Kanuk, M.J. (1972).-«Sterol additives as polymerization inhibitors for frying oils".-J. Am. Oil Chemists' Soc. 49, 298-301.

Skrodski, M., Olszewski, Z., Gorska, Z., Maj, J., Kujawa, E.K. y Wesolowska, D. (1982).-«Method of making preserved fish».-Pol. PL. 129,407.

Tanaka, Y. y Tahashi, E. (1980).- «Fresh meat preservation by external chemical treatment».-Jpn. Kokai Tokkyo Koho JP 80 16,742.

Takeda Industries Ltd. (1983).-«Food preservative composition».-Jpn. Kokai Tokkyo Koho JP 58,111,669.

Tanabe, S. (1982).-«Food antioxidant composition».-Jpn. Kokai Tokkyo Koho JP 59 15,577.

Tanaka, K. y Tahashi, E. (1980).-«Fresh meat preservation by external chemical treatment".-Jpn. Kokai Tokkyo Koho JP 80 16,742.

Tanaka, K., Yoshida, T. y Kasai, Z. (1974).-«Distribution of mineral elements in the outer layer of riz and wheat grains using electron micro probe $X$ ray analysis".-Soil Sci. Plant Nutr. 20, 87-91.-C.A. 81, 1298n.

Tanikawa, E., Motohiro, T., Shimizu, H. y Matsuda, T. (1972).-«Phytic acid as food preservative".-Jpn. Kokai Tokkyo Koho JP 71 13,667.

Uchino, K. (1987).-«Preservatives for fresh foods».-Jpn. Kokai Tokkyo Koho JP 62,198,372.

Uchino, K., Nagawa, M., Tonosaki, Y., Oda, M. y Fukuchi, A. (1988).-«Kojic acid as an anti-speck agent».-Agr. Biol. Chem. 52, 2609-2610.

Uekoki, T., Maedo, M. y Kadota, G. (1977).-«Color development in caviar».Jpn. Kokai Tokkyo Koho JP 77 70,048.

Ueno, S. y Ohmura, Y. (1974).-«Processing fresh Knehneromyces nameko for storage"..-Jpn. Kokai Tokkyo Koho JP 73 10,223.

Ueno, S., Uhtake, O. y Suzuki, Y. (1978).-«Darkening prevention in egg processed food".-Jpn. Kokai Tokkyo Koho JP 77 125,668.

Van Doorne, H., Scheffer, W.A., Hadiutomo, M. y Van der Bosch, E. (1985).«Microbial contamination of a vitamin A formulation prepared in local pharmacia, and its preservation against yeasts and molds".-Antoine van Leeuwenhock 50, 405-416.-C.A. 102, 137771w.

Vohra, P., Gray, G.A. y Kratzer, F.H. (1965).-«Phytic acid-metal complexes».-Proc. Soc. Exptl. Biol. Med. 120, 447-449.-C.A. 64, 6069f.

Wakita, K. (1977).-«Cooked noodle preservative».-Jpn. Kokai Tokkyo Koho JP 7790,642 .

Watanabe, K. (1987).-«Water-soluble food preservative powders containing chitosan and lactic acid (salts) or acetic acid (salts)".--Jpn. Kokai Tokkyo Koho JP 01 95,756

Wichlack, H. y Balcerek, H. (1986).-«Improving the storage stability of beef carcass quarters by lactic acid spraying".-Med. Weter. 42, 540-541.C.A. $106,137175 \mathrm{n}$.

Williamson, E. (1988).-«The antioxidant activity of $\Delta^{5}$-avenasterol».-Univ. Readiing. U.K.

Yamamota, A., Morita, H. y Shimizo, R. (1973).-«Lipid containing food».-Jpn. Kokai Tokkyo Koho JP 73 16,181.

Yi, B.H. y Kim, D.H. (1982).-«Antioxidant activity of maltol, kojic acid, levulinic acid, furfural, 5-hydroxymethylfurfural, and pyrazine».-Hangukm Sikp'um Kwahakhoe Chi. 14, 265-270.-C.A. 98, 3666f.

Yokohama, H., Kakehi, K., Ozaki, H. y Hayashi, Y. (1988).-«Stabilized alkylene oxide adducts".-Ger. Offen. DE 3,604,035.

Zhang, Q. y Lin, X. (1986).-«Experimental studies on the prevention of blackening of frozen prawns".-Fujian Shifan Daxue Xuebao Ziran Kexueban 2, 109-112.-C.A. 107, 76330f.

(Recibido: Junio 1992) 